\title{
Practice and Evaluation of Pre-prescription Review Mode
}

\author{
Zhaoshuai Ji ${ }^{\dagger}$, Weiwei Song ${ }^{\dagger}$, Xu Yan, Chao Ai ${ }^{*}$ \\ Department of Pharmacy, Beijing Tsinghua Changgung Hospital Affiliated to Tsinghua University, Tsinghua University Clinical Medical \\ College, Beijing, China
}

\section{Email address:}

jizhaoshuai@btch.edu.cn (Zhaoshuai Ji),swwa02163@btch.edu.cn (Weiwei Song), yxa00412@btch.edu.cn (XuYan), aichao@btch.edu.cn (Chao Ai)

${ }^{*}$ Corresponding author

${ }^{\dagger}$ Zhaoshuai Ji and Weiwei Song are co-first authors.

\section{To cite this article:}

Zhaoshuai Ji, Weiwei Song, Xu Yan, Chao Ai. Practice and Evaluation of Pre-prescription Review Mode. Pharmaceutical Science and Technology. Vol. 3, No. 1, 2019, pp. 1-6. doi: 10.11648/j.pst.20190301.11

Received: January 28, 2019; Accepted: March 11, 2019; Published: May 11, 2019

\begin{abstract}
OBJECTIVE: To increase awareness of pre-prescription review and promote the development of its mode. METHODS: The pre-prescription review and the traditional prescription review were compared from the process. The 1239 of irrational prescriptions in outpatient and emergency clinics from June 2016 to June 2017 were analyzed. RESULTS: Compared with the traditional prescription review, the pre-prescription review had the advantages of reducing the waiting time for medicine, decreasing the inappropriate prescription, simplifying the returned prescription processes, etc. However, there were still some problems, such as poor auditability of non-standard prescription, low audit efficiency at rush hour, false-negative and false-positive prescription, etc. Among the 1239 irrational prescriptions in our hospital, the main factors were incomplete clinical diagnosis, inappropriate use and dosage, no indication of disease, inappropriate dosage forms or routes of administration. CONCLUSION: The pre-prescription review can ensure rational use of drugs and safe medication in clinic.
\end{abstract}

Keywords: Pre-prescription Review, Traditional Prescription Review, Rational Use of Drugs, Irrational Prescription

\section{Introduction}

With the promotion of deepening medical reform in Beijing in 2017, Pharmacy had changed from benefit department to cost department. However, medical reform put forward higher requirements for pharmacists. Strengthened drug prescription review and prescription review to promote rational drug use. This prompted the transformation of the pharmaceutical sector from a drug supply guarantee department to a drug safety guarantee department. The direction for future pharmacy staff to work together will be to explore new mode of pharmaceutical service and promote the transformation of pharmacists. [1, 2] The number of people killed in medical malpractice in the United States is between 44,000 and 98,000. More than 7,000 patients died of "medication errors", mainly prescription problems and drug delivery errors. [3] The deaths of more than 2.5 million patients in approximately 50 million hospitalized patients each year in China are related to drug-induced adverse reactions. [4] Clinical irrational use of drugs often brings inevitable pain or even death to patients. [5,
6] The pre-prescription review was a new model for hospital in recent years, which was more conducive to the safety of patients and the rational use of drugs compared with the traditional prescription review. [7] However, the pre-prescription review was still in the initial stage of development, and there were relatively few domestic reports. The purpose of this paper was to summarize the structure, process and characteristics of the pre-prescription review, and to share the problems and experiences in the practice of the new model, so as to improve the understanding of the pre-prescription review.

\section{Materials and Methods}

\subsection{Sample}

A total of 1,239 irrational prescriptions were found from June 2016 to June 2017. We selected them from the pre-prescription review of emergency pharmacies, outpatient clinics and children's pharmacies. 


\subsection{Analytical Method}

The irrational prescriptions were classified according to the requirements of the prescription management method issued in 2007 and the hospital prescription review management specification (trial) issued in 2010. [8, 9] We used descriptive statistics to calculate and analyzed Pareto charts for the components of irrational prescriptions. According to the classification principle of Pareto chart, the cumulative composition ratio was between $0 \%$ and $80 \%$ as the main factor, which was defined as category A, $80 \%$ to $90 \%$ as the secondary factor, category $\mathrm{B}, 90 \%$ to $100 \%$ as the general factor and category $\mathrm{C}$.

\subsection{Drawing of the Pareto Chart [10-12]}

The histogram was drawn with the irrational prescription problem as the abscissa, and the irrational prescription number was the ordinate; the Pareto chart was drawn with the irrational prescription problem as the abscissa and the cumulative composition ratio was the ordinate.

\section{Result}

\subsection{Statistical Data}

There were 1,239 cases of irrational prescriptions, including 164 cases of supernormal prescriptions, 498 cases of improper prescriptions, and 577 cases of irregular prescriptions. The specific statistics were shown in Table 1.

Table 1. Irrational prescription statistics.

\begin{tabular}{lll}
\hline Classification & Number of cases & Proportion\% \\
\hline Irregular prescriptions & 577 & 46.6 \\
Improper prescriptions & 498 & 40.2 \\
Supernormal prescriptions & 164 & 13.2 \\
Total & 1239 & 100 \\
\hline
\end{tabular}

\subsection{Pareto Chart}

The various factors constituting the irrational prescription were graded and classified. The results were shown in Table 2. The irrational prescription type was the abscissa, and the Pareto chart was drawn with the number of irrational prescriptions and the cumulative percentage as the ordinate. The results were shown in Figure 1. From the results in Table 2 and Figure 1, it could see that the main components of the irrational prescription were incomplete clinical diagnosis, improper usage and dosage, no indication medication, pharmaceutical dosage form or Improper way to take medication. [13]

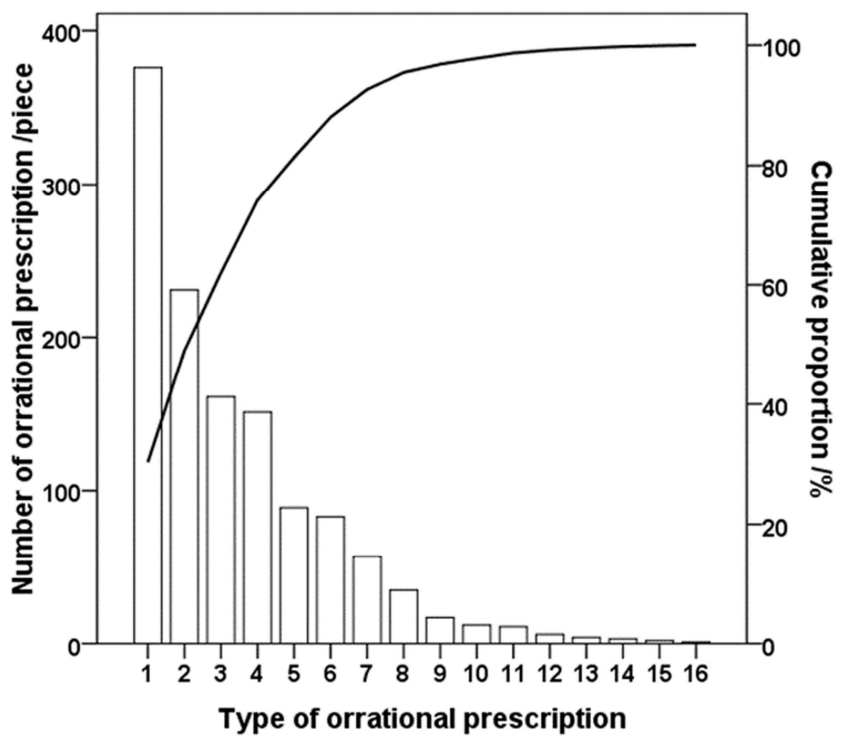

Figure 1. Pareto diagram on irrational prescriptions.

Table 2. Factor analysis of irrational prescriptions.

\begin{tabular}{|c|c|c|c|c|c|}
\hline Sequence & Type & $\begin{array}{l}\text { Number of } \\
\text { prescriptions } \\
\text { (sheet) }\end{array}$ & $\begin{array}{l}\text { Composition } \\
\text { ratio }(\%)\end{array}$ & $\begin{array}{l}\text { Cumulative } \\
\text { percentage }(\%)\end{array}$ & $\begin{array}{l}\text { Factor } \\
\text { classification }\end{array}$ \\
\hline 1 & Incomplete clinical diagnosis & 376 & 30.3 & 30.3 & A \\
\hline 2 & Improper usage and dosage & 231 & 18.6 & 49.0 & A \\
\hline 3 & No indication medication & 161 & 13.0 & 62.0 & A \\
\hline 4 & Pharmaceutical dosage form or Improper way to take medication & 151 & 12.2 & 74.2 & A \\
\hline 5 & $\begin{array}{l}\text { The doctor did not prescribe antibiotics according to the regulations on } \\
\text { the clinical application of antibacterial drugs }\end{array}$ & 89 & 7.2 & 81.4 & B \\
\hline 6 & Other medications are not suitable & 83 & 6.7 & 88.1 & B \\
\hline 7 & Incompatibility or adverse reaction & 57 & 4.6 & 92.7 & $\mathrm{C}$ \\
\hline 8 & Selection of drugs is not suitable & 35 & 2.8 & 95.5 & $\mathrm{C}$ \\
\hline 9 & $\begin{array}{l}\text { Physician signature, signature chapter is not standardized or } \\
\text { inconsistent with signature and signature chapter }\end{array}$ & 17 & 1.4 & 96.9 & $\mathrm{C}$ \\
\hline 10 & $\begin{array}{l}\text { Drugs with special management have not implemented relevant } \\
\text { national regulations }\end{array}$ & 12 & 1.0 & 97.8 & $\mathrm{C}$ \\
\hline 11 & Combination therapy is not suitable & 11 & 0.9 & 98.7 & $\mathrm{C}$ \\
\hline 12 & Inappropriate indications & 6 & 0.5 & 99.2 & $\mathrm{C}$ \\
\hline 13 & Prescription color is not suitable & 4 & 0.3 & 99.5 & $\mathrm{C}$ \\
\hline 14 & Repeat drugs & 3 & 0.2 & 99.8 & $\mathrm{C}$ \\
\hline 15 & $\begin{array}{l}\text { There is no valid reason to prescribe two or more drugs with the same } \\
\text { pharmacological effects at the same time for the same patient }\end{array}$ & 2 & 0.2 & 99.9 & $\mathrm{C}$ \\
\hline 16 & $\begin{array}{l}\text { Incomplete prescription information } \\
\text { Total }\end{array}$ & $\begin{array}{l}1 \\
1239\end{array}$ & $\begin{array}{l}0.1 \\
100\end{array}$ & 100.0 & $\mathrm{C}$ \\
\hline
\end{tabular}




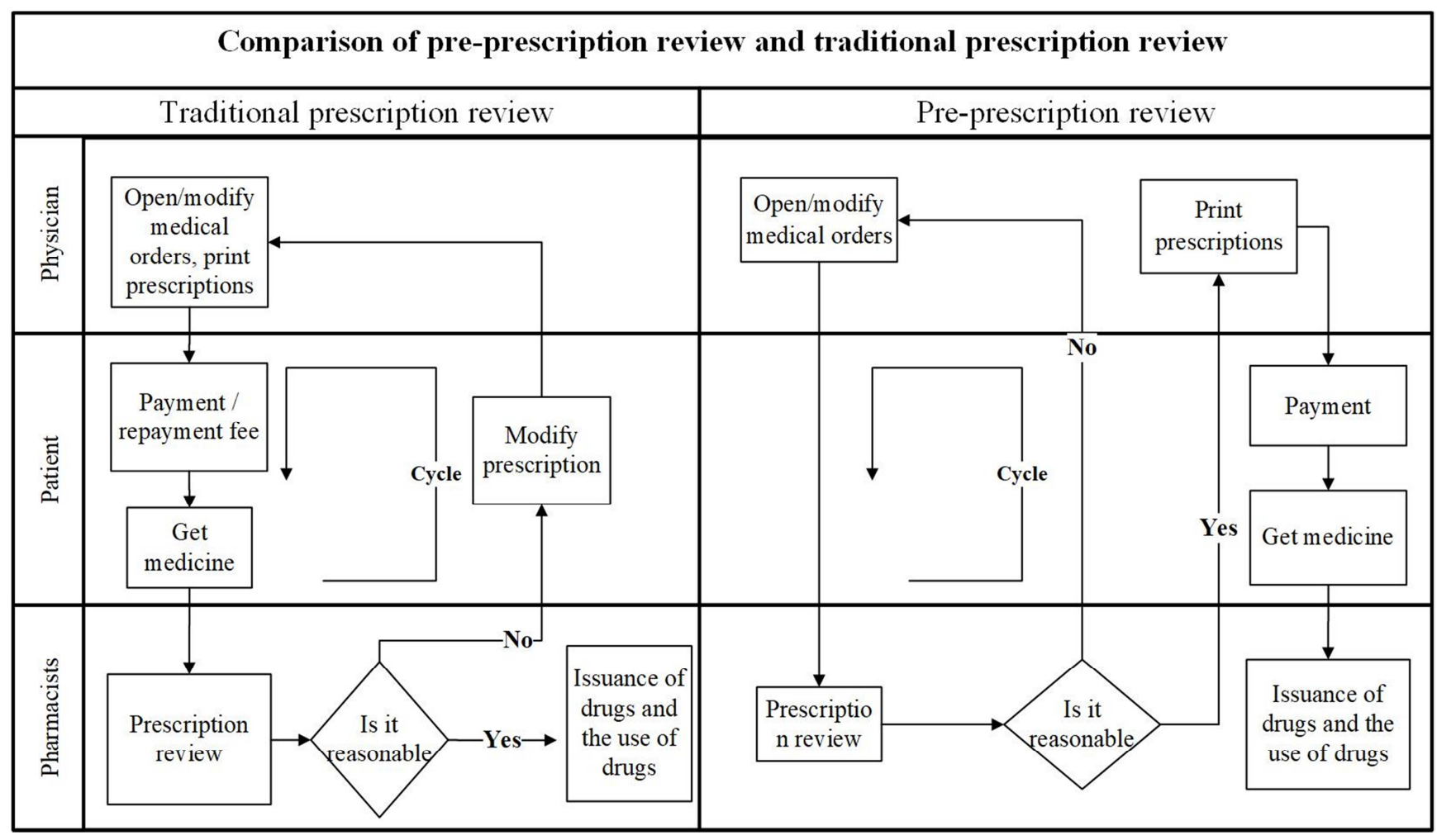

Figure 2. Comparison of pre-prescription review and traditional prescription review.

\section{Discussion}

This paper compared the traditional prescription review with the pre-prescription review through the process and the main division of labor, as shown in Figure 2. The pre-prescription review was to pre-subscribe the prescription review time before the payment and pharmacy drug-requiring linked, and completed the prescription review work before the patient paid the fee. The approved prescription print was delivered to the patient to complete the follow-up payment and drug collection. Compared with the traditional mode, its characteristics were as follows:

\subsection{Reduce the Time to Receive Medicines}

As can be seen from Figure 2, the patient only needed to go through the two steps of payment and drug collection to obtain the required drugs. The new review reduced the time required for patients to travel to and from doctor's office and payment offices and pharmacies multiple times due to irrational prescriptions in the traditional review, while also reducing the waiting time for each counter. [14, 15]

\subsection{Irrational Prescription Modification Process Was Simplified}

The pre-prescription review was done by means of rational drug software supported by big data and by reviewing pharmacists. The hospital information system would conduct a preliminary review and screening of the wills opened by the doctors, and the rational wills would directly print the prescriptions. The irrational prescriptions would be sent to the auditing pharmacist for further review, and the rational prescriptions would be printed after the pharmacists review. For irrational medical advice, the pharmacist communicated with the doctor and the doctor revised the prescription. [16] The whole process of irrational medical order modification was completed by doctors and pharmacists by means of hospital information system or telephone communication, and did not require patient participation. [17]

\subsection{Continuously Personalized and Rational Drug Use System}

The operation of rational drug software required the support of big data drug information, including: usage and dosage, adverse reactions, incompatibility, interaction and so on. The data of the software was updated regularly, and the data update came from the guide and expert consensus. This source belonged to the common data. However, a qualified prescription review software should be able to support the user to personalize the configuration as needed, such as information on the instructions, medication information for clinical trials, and rational medication information after clinical demonstration. As time goes by, the rational drug software would be continuously updated and improved, and it would gradually merge with the hospital. [18-20]

\subsection{Reduced Number of Prescriptions Irrational for Medication}

The main factors constituting the irrational prescription in the pre-treatment review of the hospital incomplete included clinical diagnosis, improper usage and dosage, 
non-indications, pharmaceutical dosage form or improper way to take medication. Similar studies of previous traditional prescription review had shown that the main factors of irrational prescriptions included inappropriate selection of drugs, unsuitable combination of drugs, improper usage and dosage, and failure to prescribe according to antibacterial drug management regulations. $[10,21]$

The pre-prescription review was based on the rational use of the drug software. Most of the drugs were not suitable for intervention and reminder, when the doctor prescribed the patient. At the same time, the software could provide doctors with information on the instructions for the use of drugs and the compatibility of drugs. Therefore, after applying the pre-prescription review, the relative number of prescriptions for irrational prescriptions was reduced, and the irregular prescriptions and super-prescriptions were relatively increased. [22]

\subsection{Increase the Comprehensive Ability of Pharmacists}

Under the new prescription review, the pharmacy needed to increase the prescription review pharmacist's job position. The content of the work was mainly for the rationality of reviewing prescriptions or medical orders. The rational drug use system would send irrational prescriptions or medical ordered to the auditing pharmacist, and the auditing pharmacists would judge the rationality according to their professional ability and hospital policy. Irrational prescriptions needed to be promptly fed back to the doctor who opened the doctor. [23] The development of the pre-prescription review required reviewing the comprehensive ability of the pharmacist to have professional ability, learning ability and communication ability. The audit pharmacist was also a direction for the transformation of pharmacists under the new medical reform form.

\section{Problems and Thoughts in the Implementation of Pre-prescription Review}

\subsection{Ability to Review Non-standard Prescriptions}

The printed prescription did not have the doctor's signature and seal, or the signature seal did not match the paper signature retention sample and the color of the prescription paper was wrong. This problem occurred after the pre-prescription. The hospital system was still unable to review and control. In the future, we will be able to effectively reduce the occurrence of irregular prescriptions by improving the function of the hospital system and increasing the use of prescription color reminder and the implementation of electronic signatures.

\subsection{Peak Review of Prescription Efficiency}

Irrational prescriptions would increase proportionally during peak hours, and if the results of the review could not be promptly reported back to the doctor, the waiting time for doctors and patients would increase. Now it set the time $\mathrm{T}$ value for the pharmacist to review the prescription after the doctor submit the prescription, and print directly over the $\mathrm{T}$ value. A prescription that had not been reviewed within the specified time period was a traditional review. The key to solving the problem at present is the reasonable setting of the $\mathrm{T}$ value and the manpower optimization configuration of the audit pharmacist.

\subsection{Irrational Prescription for False Negatives and False Positives [24]}

The occurrence of false negatives and false positives results might be due to incomplete or untimely updated of rational medication information. False negative irrational prescriptions increased the risk of medication for patients. False positive prescriptions increased the unnecessary workload of pharmacists and reduced the efficiency of reviewing prescriptions. In view of the above problems, the current measures can be adopted: 1. Regularly carry out prescription review work, screen out false negative and unreasonable prescriptions, and then reduce false negative irrational prescriptions through information system intervention or drug committee notification, etc. 2. Establish professional information pharmacist team is engaged in the search and finishing of pharmaceutical information, as well as the maintenance of rational drug software information to ensure timely updating of drug information.

\subsection{Review the Differences in the Professional Level and Subjective Judgment of Pharmacists}

The speed and quality of prescription review were influenced by factors such as the pharmacist's professional ability, knowledge reserve, objective judgment criteria, and subjective judgment criteria. [25, 26] Standardized training and assessment were established within the pharmacy department, and the pharmacist could pass the examination before becoming an audit pharmacist. In addition, according to the different professional fields that pharmacists were good at, it was possible to set up different professional auditing pharmacists, such as cardiology, endocrinology, pediatrics, obstetrics and gynaecology to guide pharmacist s to carry out deeper learning. When the outpatient review prescription is made, the task of the prescription review can be set through the hospital information system to set the division and prescription amount, so that the resources can be optimally configured. [27-29]

\subsection{Optimal Configuration of Human Resources}

The pre-prescription review was completed by means of the support of big data in the rational drug use software and the review of the pharmacist. The purpose of this model was to reduce the patient's drug taking time, optimized the patient's drug purchase process, and ensured the patient's medication safety. But it did not mean that the pharmacy needed to invest more manpower to participate in the work of prescription review. Pre-prescription review was in the early stages of 
development, and the reasons for the need to arrange more pharmacists in the process of reviewing prescriptions include: the problem of the rational use of the software itself, the mismatch between the database and the medication habits of the hospital, and the lack of software and processes for doctors and pharmacists. Familiar, pharmacist's lack of professional ability, peak medication, etc. Our hospital had increased the rational use of drug software, database resources, increased knowledge of pharmacists and the degree of cooperation with doctors, as well as the number of irrational prescriptions and feedback time. Indicators, when the indicators are stable, considered gradually developing the audit department and appropriately increasing the manpower of the audit pharmacists.

\section{Conclusion}

While reflecting the value of the pharmacist, it also made the pharmacy service more accurate, and the patient's medication was more safe and effective, under the pre-prescription review. $[30,31]$ However, the pre-prescription review was still in the initial stage of development, and there were many problems and deficiencies. Therefore, we must correctly understand and understand its characteristics, advantages and disadvantages, and constantly improve and promote the development of pre-prescription review. In the near future, the pharmacy will play an active role in ensuring safe and rational use of drugs.

\section{References}

[1] CHEN XB JX. Structuring of the hospital prescription review system. Chinese Journal of Hospital Administration. Vol. 28, No. 2, 2012, pp. 132-135.

[2] ZHU HY ZC, JIA W.. The effect of prescription review on the quality of continuous improvement of the prescription. Herald Medicine. Vol. 31, No. 4, 2012, pp. 544-546.

[3] LINDATK, JANETMC, MOLLASD. To error is human: building a safer health system [M]. WashingtonDC: NationalAcademyPress. 2000.

[4] LIAO Li-na, LI Xin, HUANG Fei, TAO Xia CW-s, WU rong. Role of the Pre-prescription Review System in Ensuring the Safety of Drug Use in Patients. Chinese Hospital Management. Vol. 38, No. 10, 2018, pp. 23-25.

[5] LEAPELL, LAIRDNM. Incidence of adverse events and negligence in hospitalized patients [J]. N Engl J Med. Vol. 324, No. 7, 1991, pp. 370-376.

[6] Bates DW, Cullen DJ, Laird N et al. Incidence of adverse drug events and potential adverse drug events. Implications for prevention. ADE Prevention Study Group. Jama. Vol. 274, No. 1, 1995, pp. 29-34.

[7] Yue F ZH, Yi ZG, Chou ZH, Zhang Z, Ji HJ. Comparison of Pre-Prescription Audit and Post-Prescription Audit in Improving Pharmaceutical Care. China Pharmaceuticals. Vol. 25, No. 12, 2016, pp. 77-78.

[8] LIN Wen-qiang LQ, ZHANG Jin, LIU Mao-bo. Establishment of automatic auditing and intervention system for outpatient prescriptions in our hospital. China Journal of Hospital Pharmacy. Vol. 36, No. 11, 2016, pp. 943-946.

[9] LI Xin LL-n, CHEN Yan-hong, TAO Xia, CHEN Wan-sheng, WU Rong. Application of prescription pre-checking system in checking of outpatient prescriptiongs. Practical Pharmacy And Clinical Remedies. No. 4, 2018, pp. 475-479.

[10] DONG ZQ ZJ, LUO YM, ZHANG GL, YANG Y. Pareto Chart Analysis of Unreasonable Factors of Prescription in Outpatient and Emergency Departments of Our Hospital during 2013-2015. China Pharmacy. Vol. 28, No. 17, 2017, pp. 2340-2342.

[11] GAO Y CQ, XING XX, JIANG DC. Analysis of irrational prescriptions in neurological mergency department in a hospital using Pareto diagram. Practical Pharmacy And Clinical Remedies. Vol. 19, No. 07, 2016, pp. 898-900.

[12] LIANG JH LP, ZHANG W, CHEN N, ZHEN JC. Analysis of 1 223 irrational prescriptions in Beijing Jishuitan Hospital's outpatient. Clinical Medication Journal. Vol. 15, No. 03, 2017, pp. 36-40.

[13] ZHANG Dong-su ZM-m, YANG Mei, SU Ye, SHI Hong, KANG Yi, CAI Le, LI Meng, SI TU Wei, BAO Jiang-xia, ZHU Ying. Practice of outpatient prescription pre-intervention based on artificial intelligence. Clinical Medication Journal. Vol. 15, No. 12, 2017, pp. 45-48.

[14] GU ZS WW. Promoting Pre-Prescription Review to Improve Safe Medication. Hospital Management Forum. Vol. 30, No. 11, 2013, pp. 54-56.

[15] NING H WX, ZHAO J, ZHANG YH. The practice of prescriptions audit before payment by computer system. Journal of Pharmacutical Practice. Vol. 33, No. 02, 2015, pp. 176-178.

[16] XIE Yangfan ZH. Prescription Review under the New Model of Pharmaceutical Care. CHINESE MEDICINE MODERN DISTANCE EDICATION OF CHIAN. Vol. 16, No. 11, 2018, pp. 26-27.

[17] WANG X XJ, DING X, NING H. Operation Practice of Outpatient Prescriptions Audit before Payment in Our Hospital. China Pharmacy. Vol. 27, No. 04, 2016, pp. 461-463.

[18] LX G. An Application Analysis of Prescription Automatic Screening System for Drug Monitoring and Rational Use of Drug. China Licensed Pharmacist. Vol. 7, No. 07, 2010, pp. 24-27.

[19] TONG SH BY, GUO JJ, ZHU YL. Application of information-based prescription comment management system in "sunshine project". Herald of Medicine. Vol. 33, No. 07, 2014, pp. 979-981.

[20] ZHONG XJ LL, GAO YL, FU L, ZHANG JF. The Application of Rational Drug Use Monitoring System and Pharmacist Workstation in Prescriptions Checking. China Pharmacy. Vol. 25, No. 42, 2014, pp. 4010-4012.

[21] CHEN Y LL, HUANG J, FENG WY. Analysis of Pareto diagram on irrational prescription in our hospital. The Chinese Journal of Clinical Pharmacology. Vol. 31, No. 13, 2015, pp. 1325-1327.

[22] CHEN R GB, GONG YH, YU X, BAO JA. Application of Rational Drug Use Software System in the Drug Dispensing in Outpatient Department of Our Hospital. China Pharmacy. Vol. 27, No. 22, 2016, pp. 3091-3094. 
[23] Feng L. Analysis of intervention on irrational prescriptions in outpatient pharmacy. Journal of Pharmaceutical Research. Vol. 35, No. 05, 2016, pp. 303-304.

[24] ZHU FF SY, LIAO LN, TAO X, CHEN WS, WU R. Application and Practice of Intelligent Management and Solution for Clinical Rational Drug Use in Our Hospital. China Pharmacy. Vol. 27, No. 25, 2016, pp. 3528-3531.

[25] LY L. Discussion on Difficult Points and Judgement Standard in Carrying out Hospital Prescription Review Management Specification (Trial). Evaluation and Analysis of Drug-Use in Hospitals of China. Vol. 16, No. 01, 2016, pp. 107-110.

[26] ZHAO HQ LP. Thoughts and Suggestions on Unreasonable Prescription Criteria for Prescription Comment. Chinese Journal of Pharmacovigilance. Vol 9., No. 06, 2012, pp. 367-369.

[27] LIU R LJ, DU FX, LING HH, ZHU SW, WANG SL. Common Mistake and Coping Strategies of Performance Management in Hospital Pharmacy. China Pharmacy. Vol. 26, No. 01, 2015, pp. 83-86.
[28] LIU Z YJ, TAO X, YAO JY, TAO X, CHEN WS. Analysis of Inpatient Medical Orders Review in Our Hospital in 2013. China Pharmacy. Vol. 26, No. 05, 2015, pp. 599-601.

[29] LV XF ZD, BIAN YX. Exploration of Carrying out Medication Order Review and Pharmaceutical Care System in Inpatient Pharmacy. China Pharmaceuticals. Vol. 25, No. 20, 2016, pp. 86-88.

[30] LI J CW, ZHANG LC, BIAN YF, WANG ZZ. Promotion of rational drug use through prescription evaluation and review by clinical pharmacists in outpatient department. Pharm Care Res. Vol. 15, No. 01, 2015, pp. 18-21.

[31] LIN WL CY. Analysis on the pre-Intervation of irrational prescription in outpatient department. China Journal of Hospital Pharmacy. Vol 32., No. 05, 2012, pp. 382-384. 